\title{
In Vivo Analysis of Bone Strain about the Sagittal Suture in Macaca mulatta during Masticatory Movements
}

\author{
Rolf G. Behrents, David S. Carlson, and Thomas Abielnour* \\ Center for Human Growth and Development and *Department of Anatomy, The University \\ of Michigan, Ann Arbor, Michigan 48109, USA
}

In vivo strain gauge analysis demonstrated that tensile bone strain is transmitted along the cranial vault to the parasagittal region during isotonic temporalis contraction. This strain is sufficient to cause measurable separation of the sagittal suture, and thus could influence growth at the sutural margins.

\section{J Dent Res 57(9-10):904-908, Sept.-Oct. 1978}

Early views of craniofacial growth regarded the increase in size and change in shape and position of the bones of the cranial vault to be the result of ectocranial deposition and endocranial resorption, with the sutures playing little or no role in the increase in skull size. ${ }^{1,2}$ Later views held that the cranial vault grows actively at the sutures. While this has remained the generally accepted view through the present day, disagreement remains concerning the local control mechanisms affecting sutural growth, i.e., the source of the force which causes cranial bones to move apart during growth and initiate bone formation at the sutural margins.

Weinmann and Sicher ${ }^{3}$ proposed that an intrinsically regulated proliferation of sutural tissue is the driving force for the separation and growth of the cranial bones. The works of Moss, ${ }^{4}$ Girgis and Pritchard ${ }^{5-7}$ and Young, ${ }^{8}$ however, indicate that the separating force is largely the result of the expansive growth of the brain which separates the cranial bones and results in compensatory osteogenic deposition at the sutural margins. Another opinion held that the growth of the cartilaginous synchrodoses of the cranial base provides the force

Received for publication August 30, 1977.

Accepted for publication February 1, 1978.

This research was supported in part by Postdoctoral Research Fellowship DE-05104 and DE-05101 from the National Institute of Dental Research, National Institutes of Health. which causes a separation of the cranial bones and compensatory osteogenic activity at the sutures. ${ }^{9}$

The influence of muscle forces in determining the morphology of the cranial vault has been thought to be relatively minor, and has been discussed primarily in terms of the production of secondary osseous formations (i.e., crests, ridges, etc.) ${ }^{9-11}$ Perhaps as a result of the emphasis on cerebral expansion as a primary cause of cranial growth and on muscle function as a cause of secondary osseous formation, however, very little attention has been directed toward the role of muscular forces in affecting the growth and resultant morphology of the sutures. Those studies which have been done have been largely theoretical, and indicate that stresses generated within the craniofacial complex are reflected in and resisted by various morphological configurations of sutures. ${ }^{12-14}$.

W th this in mind, it is probable that the suture system within the mammalian cranial vault functions, at least in part, to resist tensile stresses related to mastication. Furthermore, given the generally accepted view that separation of the sutures may stimulate compensatory osteogenic activity, it is possible that separation of the sutures, as a result of mastication, may actually stimulate sutural growth. The purpose of this analysis was to determine the influence of the temporalis muscles during the biting action on the cranial vault in growing rhesus monkeys (Macaca mulatta). Specifically, we sought to determine (1) the nature of the stresses about the region of the sagittal suture in vivo during molar biting, and (2) whether these forces are sufficient to cause a separation of the parietal bones at the sagittal suture. Such a twofold approach permitted us to distinguish between bone strain and movement in the sagittal region. 


\section{Materials and Methods}

Single-element foil strain gauges were used to measure in vivo bone strain along the sagittal suture in response to the simulation of the biting action by the temporalis muscles. Additionally, relative bite pressure was monitored in order to assess the force produced by the temporalis muscle relative to the stresses transmitted along the cranial vault.

SAmpLE.-Two juvenile (as judged by dental criteria: 2 years) Macaca mulatta were used as models for this experiment. Young, growing rhesus monkeys were used in order to insure that there was no fusion of the sagittal suture and that the potential for sutural separation remained.

Strain gauge placement. - Single-element strain gauges* were attached (1) parasagittally on the right side of the sagittal suture on one animal, and (2) across the sagittal suture on the other. The procedure used for strain gauge placement was similar to that recently described by Hylander. ${ }^{15}$ The animal was anesthetized, $\uparrow$ and, following infiltration of the area with a local anesthetic, $\$$ a $3-\mathrm{cm}$ incision was made along the midline of the cranium in the region of the anterior one-third of the sagittal suture. The soft tissues and the periosteum were stripped from the bone and reflected to expose the underlying cortical bone and suture. After achieving hemostasis, the bonding site was degreased with a solution of 2 parts chloroform and 1 part methanol and then dried with acetone. The bonding site was then flooded with surface activator and the adhesive§ was applied to the underside of the gauge. The gauge had previously been coated with a waterresistant plastic coating $\#$ on the remaining surfaces. The gauge was then pressed in the ap-

* Type EA-08-030LB-120, Micromeasurements, Romulus, Mi.

† Sernylan (Phencyclidine, HCL), Bio-ceutic Lab, Inc., St. Joseph, Mo. dose: $1.0 \mathrm{mg} / \mathrm{Kg}$; Sodium Pentobarbital dose: $5 \mathrm{mg} / \mathrm{Kg}$.

\$ Lidccaine HCL, $2 \%$ with epinephrine $1: 100,000$, Elkins-Sinn, Cherry Hill, NJ.

$\S$ M-Bond 200 adhesive, Micromeasurements, Romulus, Mi.

\# White silicone bathtub calk, General Electric, Waterford, NJ.

II Model 7 Polygraph with driver amplifier 7 DAC, Grass Instruments Company, Quincy, Ma.

** Model E2B subdermal electrode, Grass Instrument Company, Quincy, $\mathbf{M a}$.

t† SD5 stimulator, Grass Instrument Company, Quincy, Ma.

\$ Model P37 Physiological pressure transducer, Stratham Instrument Company, Oxnard, Ca.

$\S \S$ Model 350-1100B Carrier preamplifier, Sanborn, Waltham, Ma.

\#\# Tektronics, Portland, Or. propriate location with its long axis perpendicular to the suture, and was held under heavy finger pressure for 2 minutes. The strain gauge was connected to form one arm of a wheatstone bridge by connecting it to a bridge-amplifier device, and then was connected to the polygraph recorder. After the bridge was balanced, manual pressures were applied to the cranium until it was determined that the recording apparatus was responding properly.

Muscle stimulation.-Electrodes ${ }^{* *}$ were implanted bilaterally into the anterior and posterior regions of the temporalis muscles and connected to a stimulator. I $^{-}$The stimulation of the muscles was monitored by connecting the stimulator to the polygraph recorder.

BITE FORCE MEASUREMENT.-Relative bite pressure was also monitored in one animal to assess the relative force produced by the temporalis muscles during stimulation. This was accomplished by placing water-filled flexible tubing across the molar area of the monkey. The tubing was connected to a pressure transducert which was then connected to an amplifier, $\$ \S$ the polygraph recorder and oscilloscope.\# The pressure exerted on the transducer was calibrated using a water-filled manometer by applying known amounts of force to the transducer.

RECORDING PROCEDURE.-Animals were maintained under anesthesia throughout the experiment. The temporalis muscles were stimulated bilaterally to record both single twitch and tetanic activities. Twitch characteristics were recorded using 7 to 9 volts of electricity for a 10 millisecond duration. Tetanus was recorded using nine volts at 20 pulses per second for durations of up to 30 seconds. Strain gauge deviations, bite force pressure and excitation voltage, frequency and duration were recorded simultaneously on the polygraph recorder. Following recording sessions of approximately one hour, the protective coating was removed from the strain gauge and the location of the resistance arms of the strain gauge were observed under a dissecting microscope. The strain gauge was then removed, the tissues were sutured, penicilin was administered, and the animals were returned to the cages.

Two recording sessions were accomplished on separate animals: (1) parasagittally, approximating sagittal suture in order to measure bone strain and (2) across the sagittal suture in order to record movement between cranial bones. 


\section{Results}

Tensile strain $\llbracket$ was found to increase about the sagittal suture during isotonic single twitch and tetanic contraction of the temporalis muscles.

ParasagitTal bone strain (Fig 1),-A series of single twitch stimuli, each approximately one second apart, produced no recordable deviations. However, with tetanic stimulation, parasagittal bone strain rose to a peak of approximately 700 microstrain at maximum deflection. In each case, maximum strain occurred near the end of the tetanus. A slight compressive strain was noted briefly between the two episodes of tetanus. This may have been due to an overcompensation of the bone in its return to the original condition following cessation of muscle activity.

Transsagittal strain (Fig 2).--Single twitch stimuli of the temporalis muscles resulted in approximately 20 microstrain when the strain gauge recorded from across the sagittal suture. Strain rose definitively with each twitch, and decreased slightly prior to the onset of the next isotonic contraction.

Tetanus resulted in a marked increase in tension to a peak of approximately 180 microstrain. As expected, the soft tissue of the suture demonstrated a higher strain than bone. Bite force was equivalent to a deflection of $12.5 \mathrm{~mm}$ $\mathrm{H}_{2} \mathrm{O}$ at the initiation of tetanus, but then dropped to approximately $2 \mathrm{~mm} \mathrm{H}_{2} \mathrm{O}$. II Bite force returned to zero coincident with cessation of muscle activity, however tensile strain across the suture decreased only gradually, and was still present for a considerable time after the

1 Strain $(\mu)$ is the quotient of the change in length of an object divided by its original length. One microstrain $(\mu \varepsilon)$ equals $1 \times 10^{-6}$ units/unit (e.g., inch/inches, millimeter/millimeters, etc.).

II The force $(F)$ generated against a load by a given muscle can be represented most simply by the formula

$$
F=T+(m a)
$$

where $T=$ torque (muscle force $\times$ moment arm), and $m=$ mass and $a=$ acceleration of the lever arm. $T$ and $m$ can be considered constants in this study, while $F$ can be taken to represent the bite force. Contractions of the temporalis muscles during single twitch stimuli were isotonic, i.e., they resulted in an acceleration of the mandible. The same conditions obtained at the initiation of tetanic stimulation; however, once the jaws closed on the transducer for more than a brief instant the effect of $a$ was reduced to 0 as an isometric state of contraction was maintained for the duration of tetanic stimulation. Thus, the difference between the recorded bite forces during single twitch stimulation, the onset of tetanic stimulation and the major portion of tetanic stimulation is due to change in the acceleration of mandibular elevation during isotonic and isometric contraction.

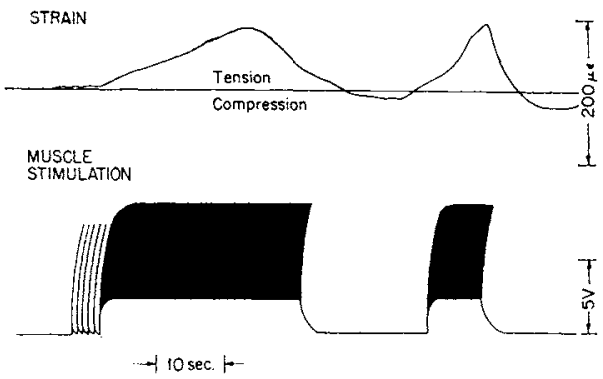

Fig. 1.-Bone strain recorded from the parasagittal region of the anterior one third of the left parietal bone in a juvenile rhesus monkey, in response to bilateral stimulation of the temporalis muscles.

tetanus was completed. This indicated that the parietal bones pulled apart at the sagittal suture during tetanic stimulation of the temporalis muscles, and that they steadily, but slowly, returned to their original relationship following cessation of function. This return, or recovery, is indicative of soft biological material and is a viscoelastic phenomenon.

\section{Discussion}

Although cranial sutures have been

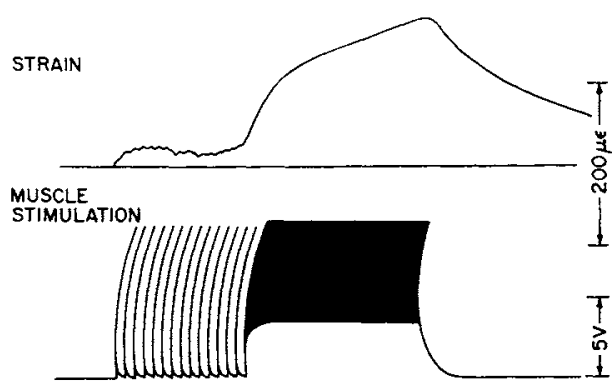

BITE
FORCE

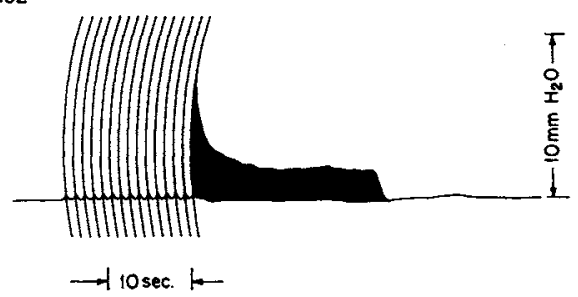

FIg 2.--Transsutural strain and relative bite force recorded from across the sagittal suture in a juvenile rhesus monkey, in response to bilateral stimulation of the temporalis muscles. III 
thought to be primarily tension-adapted systems, ${ }^{16,14,17}$ there has been no previous empirical analysis of biomechanical strains transmitted about the sutures of the vault in response to in vivo muscle function. Herring's ${ }^{14}$ analysis of cranial sutures in suids dealt only with hypothesized stress trajectories in dry skulls of adults. Experimental strain gauge studies by Endo ${ }^{18,19}$ were conducted on dry skulls of humans and a gorilla using reconstructed muscles to simulate masticatory function. Endo's studies did not extend to the sutures of the cranial vault, but focused on the facial skeleton. $\mathrm{Re}$ cent studies by Hylander ${ }^{15}$ of in vivo bone strains in the mandible of adult Galago crassicaudatus, however, have demonstrated the efficacy of in vivo strain gauge studies for the analysis of craniofacial form.

The importance of empirical data of in vivo bone strains, especially in growing animals, is particularly acute in terms of further understanding sutural growth. Documentation that (1) tensile stresses are generated about the sagittal suture during contraction of the temporalis muscles, and (2) that, even at relatively small levels of bite force, tensile stresses capable of separating the cranial bones are present provides data supportive of recent hypotheses regarding local control mechanisms of sutural growth. Specifically, Azuma et al, ${ }^{20}$ among others, have suggested that specific organelles within the linkage zone of the sutural membrane, myofibroblasts, "participate in growth and displacement utilizing a tension rather than pressure system. The myofibroblasts . . . provide, or at least actively contribute to, biomechanical tension that produces bony displacement and creates the tension type of system required."

The data from this analysis suggest that it may be the tension exerted in the sagittal suture during temporalis function, resulting in a displacement of the parietal bones, which provides the stimulus for contraction of the myofibroblasts. Another possibility is that the stretching of the connective tissue fibers of the linkage zones simply exerts tension in the periosteal membrane along the sutural margin of each parietal bone, and that this is sufficient to stimulate osteogenesis in this region. It is significant, in this respect, that the effect of tensile strains across the suture appear to be cumulative, and that there is no immediate return to the pre-stress condition due to viscoelastic recovery following cessation of muscle stimu- lation. Single twitches produced relatively small strains across the suture; however, when the temporalis muscles were tetanized with equal voltage the strain increased steadily to a peak near the cessation of excitation well above that produced by a single twitch, following which the strain decreased only gradually. This indicates that the suture responded as a viscoelastic tissue under increasing tension, and that the parietal bones were probably "pulled" back into their original position, either by passive or active contraction of the fibers within the linkage zone. In either case, the tension exerted on the periosteal membrane within the suture should be sufficient to stimulate compensatory osteogenic activity and thus effect growth of the bones of the cranial vault. Further substantiation of these results should thus have far-reaching implications for understanding craniofacial growth, suture morphology, time of suture closure and evolutionary changes in the hominoid skull.

\section{Conclusions}

Strain gauges were placed in the parasagittal region and across the sagittal suture in living monkeys. Stimulation of the temporalis muscles resulted in a maximum tension of 100 microstrain within the parietal bone just lateral to the suture, and 180 microstrain across the suture. Even with relitively small bite force, the tensile strain was sufficient to cause a separation of the sagittal suture. It was suggested that such separation might act as a major factor in the local control of osteogenesis of the sutures.

Appreciation is extended to Dr. Carl Gans, Dr. Gary Gorniak, Mr. Richard Cobel-Geard and Mr. Elwyn Gooding for their assistance in this project. We are also grateful to Ms. Christine McCabe and Mrs. Cheryl Wyman for their editorial assistance.

\section{References}

1. BRASh, J.C.: The Growth of the Jaws, Normal and Abnormal, in Health and Disease, Dental Board of the United Kingdom, London, 1924.

2. Bras H, J.C.: Some Problems in the Growth and Developmental Mechanics of Bone, Edinburgh Med J 41:305; 363, 1934.

3. Weinmann, J.P.. and Sicher, H.: Bone and Bones 2nd ed., Mosby, St. Louis, 1955.

4. Moss, M.L.: Growth of the Calvaria in the Rat: The Determination of Osseous Morphology, Am J Anat 94:333-362, 1954.

5. Girgis, F.G., and Pritchard, J.J.: Experimental Alteration of Cranial Suture Patterns, J Anat 90:573, 1956. 
6. Girgis, F.G., and Pritchard, J.J.: Analysis of Experimental Suture Deviation, J Anat $91: 578,1957$.

7. Girgis, F.G., and Pritchard, J.J.: Effects of Skull Damage and the Development of the Sutural Patterns in the Rat, $J$ Anat 92: 39-51, 1958.

8. Young, R.W.: The Influence of Cranial Contents on Postnatal Growth of the Skull in the Rat, Am J Anat 105:383-415, 1959.

9. ScotT, J.H.: Muscle Growth and Function in Relation to Skeletal Morphology, Am J Phys Anthropol 15:197-233, 1957.

10. Weidenreich, F.: The Brain and Its Role in the Phylogenetic Transformation of the Human Skull, Trans Am Phil Soc 31:321$442,1940$.

11. WASHBURN, S.L.: The Relation of the Temporal Muscle to the Form of the Skull, Anat Rec 99:239-248, 1947.

12. GaNS, C.: Studies on Amphisbaenids (Amphisbaenia, Reptilia) 1. A Taxonomic Revision of the Trogonophinae and a Functional Interpretation of the Amphisbaenid Adaptive Pattern, Bull Am Mus Nat Hist 119:129-203, 1960.

13. FrazeTta, T.H.: A Functional Consideration of Cranial Kinesis in Lizards, $J$ Morph $111: 287-320,1962$.
14. Herring, S.W.: Sutures-A Tool in Functional Cranial Analysis. Acta Anat 83:222$247,1972$.

15. Hylander, W.: In Vivo Bone Strain in the Mandible of Glago cassicaudatus, Am J Phys Anthrop 46:309-326, 1977.

16. Mednick, L.W., and Washburn, S.L.: The Role of the Sutures in the Growth of the Braincase in the Infant Pig, Am J Phys Anthrop 14:175-192, 1956.

17. Enzow, D.H.: Handbook of Facial Grozth. Philadelphia, W. B. Saunders, 1975.

18. Endo, B.: Experimental Studies on the Mechanical Significance of the Form of the Human Facial Skeleton, J Fac Sci 3:1-106, 1966.

19. Endo, B.: Analysis of Stresses Around the Orbit due to Masseter and Temporaiis Muscles Respectively, I Anthrop Soc Nip 78: 251-266, 1970.

20. Azuma, M.; EnLow, D.H.; Frederickson, R.G.; and GAstor, L.G.: A Myofibroblastic Basis for the Physical Forces that Prociuce Tooth Drift and Eruption, Skeletal Displacement at Sutures, and Periosteai Migration, in Determinants of Mandibular Form and Grozth, McNamara, J.A., JR. (ed.): Ann Arbor, Center for Human Growth and Development Monograph No. 4, 1975, p 203. 